PO 8475 ENHANCING THE CAPACITY OF THE LIBERIA MEDICINES AND HEALTH PRODUCTS REGULATORY AUTHORITY IN POST-MARKETING SURVEILLANCE OF IN VITRO DIAGNOSTICS

${ }^{1}$ Alexander E George*, ${ }^{1}$ Joseph N Somwarbi, ${ }^{1}$ David Sumo, ${ }^{2}$ Guillermo Martínez Pérez, ${ }^{2}$ Alfredo Mayor. 'Medicines and Health Products Regulatory Authority, Monrovia, Liberia; ${ }^{2}$ ISGlobal, Hospital Clínic - Universitat de Barcelona, Barcelona, Spain

\subsection{6/bmjgh-2019-EDC.112}

Background The Quality Control Laboratory (QCL) of the Liberia Medicines and Health Products Regulatory Authority (LMHRA) lacks capacity to assess the quality of in vitro diagnostics (IVDs). The LMHRA needs be strengthened to develop post-market surveillance (PostMS) regulations in order to fulfil its supervisory role for IVDs used in research and healthcare settings. IGORCADIA, an EDCTP-funded project of LMHRA and the Barcelona Institute for Global Health (ISGlobal) started in December 2017 with the aim of building LMHRA diagnostics assessment capacity.

Methods Project activities targeting the QCL include: the constitution of an in-house Technical Working Group and a Diagnostic Steering Committee involving national stakeholders to develop PostMS regulation; a Training Programme in Diagnostics Research (TPDxR) including a malaria diagnostics performance study as its post-TPDxR exercise.

Results The QCL is developing with its new knowledge and networks improved mechanisms to enact its supervisory mandate. QCL staff contributed to the development of guidance for Post-MS. Private sector and government stakeholders helped the LMHRA identify unlicensed premises where IVDs of presumably poor accuracy are available over the counter. Following the TPDxR, the QCL planned quality assurance to oversee the quality assessments on suspected substandard IVDs. Quality control tools, staff training requirements, standard inspection procedures, and PostMS registers and reports were re-designed in accordance with Good Laboratory Practice and guidance from the TPDxR.

Conclusion The LMHRA is strengthening its regulatory, inspection and PostMS capacities thanks to a partnership with a European research institution with expertise in malaria diagnostics development. To ensure that the Liberian population has access to safe quality diagnostics in routine healthcare provision and in future infectious diseases outbreaks, it is of utmost importance that the LMHRA has capacity to assess the accuracy of the non-WHO prequalified IVDs that are currently available outside the healthcare system, and is well-equipped to recall those IVDs identified as substandard.

\section{PO 8476 USER EXPERIENCE OF SMS REMINDERS TO TAKE MEDICATION AMONG PREGNANT AND BREASTFEEDING WOMEN LIVING WITH HIV IN KILIMANJARO, TANZANIA}

\footnotetext{
${ }^{1}$ Kennedy M Ngowi ${ }^{*},{ }^{2}$ Eusibious Maro, ${ }^{3}$ Pythia T Nieuwkerk, ${ }^{4}$ Rob E Aarnoutse, ${ }^{1,2}$ Blandina T Mmbaga, ${ }^{1,5}$ I Marion Sumari-De Boer. 'Kilimanjaro Clinical Research Institute, Moshi, Tanzania; ${ }^{2}$ Kilimanjaro Christian Medical Center, Moshi, Tanzania; ${ }^{3}$ Department of Medical Psychology, Academic Medical Center, Amsterdam, the Netherlands; ${ }^{4}$ Department of Pharmacy and with the Radboud Institute for Health Sciences, Radboud UMC, Nijmegen, the Netherlands; ${ }^{5}$ Department of International Health, Radboud UMC, Nijmegen, the Netherlands
}

10.1136/bmjgh-2019-EDC.113
Background Pregnant women living with HIV have difficulties in reaching adequate levels of treatment adherence. One way to intervene is sending reminder cues using short message service (SMS) texts. We conducted a pilot study on the use of SMS among pregnant and breastfeeding women living with HIV in Kilimanjaro, Tanzania. One objective was to investigate user experiences of SMS reminders.

Methods We enrolled adult (age 18-45) pregnant or breastfeeding women living with HIV from Kilimanjaro region, Tanzania. Women received a reminder SMS $30 \mathrm{~min}$ before usual time of intake. One hour after usual time of intake, they received an SMS asking whether medication was taken. SMSes were sent less-than-daily and randomly distributed over the week. During consultation we listened to their feedback on the system. After six-months, we interviewed women using a semi-structured exit interview.

Results Twenty-five women were enrolled. Two women were lost to follow-up. We received feedback from 18 women. Sixteen $(89 \%)$ said they were content about the SMS reminding. One said she had problems with privacy issues (6\%), but 5 specifically mentioned no privacy issues (28\%). Preliminary results of 18 exit interviews show that 16 women (89\%) had a good experience with the SMS. Fourteen women (78\%) found the content of SMS good; 2 women (11\%) said it was not good at all due to risk of unwanted disclosure. Three women (17\%) experienced stigma. Eleven women (61\%) told they were always able to reply to the SMS and $16(89 \%)$ believed it really improves adherence.

Conclusion We believe most women were satisfied with the SMS system. A few women had trouble with risks or fear of unwanted disclosure. One way to solve that, may be to send more neutral language messages. The results can be used for clinical trial design to investigate the effect on adherence.

\section{PO 8480 TECHNICAL FEASIBILITY OF SENDING SMS TO REMIND TAKING MEDICATION AMONG PREGNANT AND BREASTFEEDING WOMEN LIVING WITH HIV IN KILIMANJARO, TANZANIA}

${ }^{1}$ Kennedy M Ngowi ${ }^{*},{ }^{2}$ Eusibious Maro, ${ }^{3}$ Pythia T Nieuwkerk, ${ }^{4}$ Rob E Aarnoutse, ${ }^{1,2}$ Blandina T Mmbaga, ${ }^{1,5}$ I Marion Sumari-De Boer. 'Kilimanjaro Clinical Research Institute, Moshi, Tanzania; ${ }^{2}$ Kilimanjaro Christian Medical Center, Moshi, Tanzania; ${ }^{3}$ Department of Medical Psychology, Academic Medical Center, Amsterdam, the Netherlands; ${ }^{4}$ Department of Pharmacy and with the Radboud Institute for Health Sciences, Radboud UMC, Nijmegen, the Netherlands; ${ }^{5}$ Department of International Health, Radboud UMC, Nijmegen, the Netherlands

\subsection{6/bmjgh-2019-EDC.114}

Background Pregnant women living with HIV have difficulties in reaching adequate levels of adherence to treatment. One way to intervene is sending reminder cues using short message service (SMS) texts. We conducted a pilot study on the use of SMS among pregnant and breastfeeding women living with HIV in Kilimanjaro, Tanzania. One objective was to investigate technical feasibility of sending SMS reminders.

Methods We enrolled adult (age 18-45) pregnant or breastfeeding women living with HIV from Kilimanjaro region, Tanzania. Women received a reminder SMS $30 \mathrm{~min}$ before usual time of intake. One hour after usual time of intake, they received an SMS asking whether she took medication. The women had to reply with 'Yes' or 'No'. SMSes were sent lessthan-daily and randomly distributed over the week. We did 
descriptive analyses of sent messages, delivered messages and estimation of adherence based on messages.

Results We enrolled 25 women. In total, 4963 messages were sent of which 40 failed to be delivered (1\%). 1664 SMS were sent with a question if medication was taken, wich received an answer 1580 times (91\%). The answer was 'Yes' in 1137 cases (65\%), 'No' in 10 cases (0.6\%) and indefinable in 433 cases $(26 \%)$. The median adherence based on 'Yes'-answers was $74 \%$ [range 24-99]. If also counting the indefinable answers, the mean adherence was 100\% [range 95-100].

Conclusion Despite a few technical issues, we believe using SMS for reminder cues in Tanzania works well. The number of failed deliveries is nearly zero and women have replied to the majority of SMS. Efforts are needed to instruct women better on replying and on detecting the right answer in case of typing errors. We conclude that using SMS has potential to improve adherence and should be further investigated in clinical trials to determine the effect on adherence to treatment.

\section{PO 8481 HIGH HEPATITIS B VIRUS INCIDENCE AMONG HIV-1- INFECTED TREATMENT-NAIVE ADULTS IN BOTSWANA}

${ }^{1}$ Bonolo B Phinius*, ${ }^{1}$ Resego Bokete, 1,2Motswedi Anderson, 1,2Tshepiso Mbangiwa, ${ }^{1}$ Wonderful Choga, ${ }^{1,3}$ Sikhulile Moyo, ${ }^{1,3}$ Rosemary Musonda, ${ }^{4}$ Richard Marlink, 1,3Simani Gaseitsiwe. 'Botswana Harvard AIDS Institute Partnership, Gaborone, Botswana; ${ }^{2}$ University of Botswana, Gaborone, Botswana; ${ }^{3}$ Harvard T.H Chan School of Public Health, Boston, Massachusetts, USA; ${ }^{4}$ Rutgers University, New Jersey, USA

\subsection{6/bmjgh-2019-EDC.115}

Background Hepatitis B virus (HBV) is one of the leading causes of death worldwide despite a moderately potent vaccine. HBV prevalence has been shown to be higher in patients infected with the human immunodeficiency virus (HIV), hence increased liver-related morbidity and mortality, as well as general poor health outcomes in HIV-HBV co-infection. We estimated the HBV incidence among HIV-1-infected treatmentnaïve adults in a longitudinal cohort in Botswana.

Methods Plasma samples from 200 HIV-1C-infected treatmentnaive participants from a completed longitudinal cohort from 2004 to 2007 were screened for HBV surface antigen (HBsAg). HBsAg was assessed using Murex version 3 enzymelinked immunosorbent assay as per manufacturer's instructions at 4 timepoints, 12 months apart. We estimated HBV incidence with 95\% confidence interval (CI). Cox proportional regression method was used to estimate hazard ratios [gender, age $(\leq 35$ or $>35)$ years, $\mathrm{CD}^{+} \mathrm{T}$ cell count $(\leq 450$ or $>450)$ cells $/ \mu \mathrm{L}$ and HIV viral load suppression $(\leq 400$ or $>400)$ copies/ $\mathrm{mL}$.

Results The median age of screened individuals was 32 years [Q1, Q3; 28, 40] and 83.5\% [167/200] were female. Baseline median $\mathrm{CD}^{+} \mathrm{T}$ cell count was 466.35 cells/ $\mu \mathrm{L}$ [Q1, Q3: $380.43,605.75]$ and median HIV viral load was 13450 copies/mL [Q1, Q3: 2365, 37 400]. The HBV incidence was 3.6/ 100 person-years [95\% CI: 2.2-5.6]. There were no significant differences by gender, age, HIV viral load suppression and $\mathrm{CD} 4^{+} \mathrm{T}$ cell count.

Conclusion We report for the first time a high HBV incidence among HIV-infected adults in Botswana. HBV incidence was high in this population despite generally high CD4 + T cell counts and lower HIV viral loads. Early screening of HBV in $\mathrm{HIV}$-infected individuals is vital and should be included in the national HIV treatment guidelines.

\section{PO 8483 ASSOCIATIONS BETWEEN HIV AND OTHER STIS AMONG GAY, BISEXUAL MEN AND TRANSGENDER WOMEN IN NAIROBI, KENYA}

${ }^{1}$ Rodah Wanjiru*, ${ }^{2}$ Joshua Kimani, ${ }^{3}$ Adrian Smith. ${ }^{1}$ Partners for Health and Development in Africa, Nairobi, Kenya; ${ }^{2}$ Partners for Health and Development in Africa, Nairobi, Kenya; ${ }^{3}$ University of Oxford, Nuffield Department of Population Health, Old Road Campus, Oxford, UK

\subsection{6/bmjgh-2019-EDC.116}

Background Men who have sex with men (MSM) are a key target population for HIV prevention and control in Kenya. Although male sex workers remain the focus of research in Nairobi, HIV/STI prevalence has not been assessed among the wider MSM population since 2010. This study set out to reassess prevalence and associations of HIV and other STIs.

Methods Respondent-driven sampling recruited 618 MSM. Eligibility criteria were age $18+$, male (birth or currently), Nairobi residence and consensual oral or anal intercourse with a man in the last year. Consenting participants completed an online survey including current experience of STI symptoms. Participants tested for HIV [Determine, First Response $\left[2^{\text {nd }}\right.$ gen] and GeneXpert HIV-Qual [4 ${ }^{\text {th }}$ gen]], syphilis [RPR/ TPHA], hepatitis $\mathrm{B}$ and $\mathrm{C}$ [HBsAg and HCV ELISA], urine and rectal chlamydia and gonorrhea [GeneXpert CTNG]. Associations with prevalent HIV were assessed using multivariate logistic regression.

Results HIV prevalence was 26.4\% [22.6-30.6] including $0.5 \%$ [0.2-1.5] detected solely on 4 th gen testing. Prevalent HIV was independently associated with age, lower education, Kenyan birth, transgender identity and exclusive sex with men in the past 3 months but dependently associated with STI symptoms. Prevalence of syphilis was $0.8 \%$ [0.3-1.9]; hepatitis B 4.4\% [3.4-6.9]; hepatitis C $0.5 \%$ [0.2-1.5]. Current symptoms consistent with urethritis were reported by $6.4 \%$ [4.59.0] of participants. Prevalence of urethral GC and CT were 4.4\% [2.9-6.7] and 7.3\% [5.2-10.3] respectively. Symptoms consistent with proctitis were reported by $8.6 \%$ [6.3-11.6] of participants. The prevalence of rectal GC and CT were $13.3 \%$ [10.4-16.8] and 8.7\% [6.7-11.2] respectively. Overall, only $17.7 \%$ [9.2-31.2] of participants with urethral CT/NG and 17.8\% [10.7-28.0] rectal CT/NG were symptomatic.

Conclusion The burden of HIV among gay, bisexual and other MSM (GBMSM) remains considerably higher than other men in Nairobi, whilst the prevalence of syphilis and hepatitis C are relatively low. Chlamydia and gonorrhoea infections, particularly rectal, are common and frequently asymptomatic. Capacity of GBMSM-friendly and community-based providers to offer CT/NG screening should be prioritised.

\section{PO 8485 INTERFERON GAMMA RESPONSE KINETICS IN TUBERCULOSIS PATIENTS AND HOUSEHOLD CONTACTS IN THE GAMBIA}

${ }^{1}$ Georgetta Mbayo*, 'Mathieu Garand, 'Simon Donkor, ${ }^{1}$ Miriam Wathuo, ${ }^{2}$ Tom Ottenhoff, 'Olumuyiwa Owolabi, 'Jayne Sutherland. 'Vaccines and Immunity Theme, Medical Research Council (MRC) Unit at London School of Hygiene and Tropical Medicine, Banjul, The Gambia; ${ }^{2}$ Department of Infectious Diseases, Leiden University Medical Center, the Netherlands

\subsection{6/bmjgh-2019-EDC.117}

Background Methods which use Mycobacterium tuberculosis $(\mathrm{Mtb})$-specific antigens to measure IFN- $\gamma$ responses (IFN- $\gamma$ 